\title{
Against the proposition: all patients with advanced HIV disease should be offered rifabutin prophylaxis
}

The goal of primary prophylaxis in advanced HIV patients is to prevent opportunistic infection in order to improve the duration and quality of survival. Any such prophylactic policy should minimise cost and maximise benefit. Ideally any drug used should be well tolerated, inexpensive and not interact with other drugs. Selection of a primary prophylaxis against Mycobacterium avium complex (MAC) thus involves a variety of general and specific considerations. However, before considering a drug option is it possible to prevent infection by behavioural or environmental strategies? It would not seem so as MAC is ubiquitous in the environment being found in water, soil, food and cigarettes. ${ }^{1}$ There are some data suggesting that patients may also get infected from hospital water supplies, ${ }^{2}$ but even if true (and the evidence is circumstantial), this does not represent a major route of transmission.

There are problems with rifabutin the only licenced drug for MAC prophylaxis. These include: selection of drug resistant mutants and non-compliance, side effects and adverse drug reactions and the potential masking of tuberculosis.

\section{Drug resistance}

Drug resistance may occur on rifabutin prophylaxis by two mechanisms. In spite of taking MAC prophylaxis, patients are being continually exposed to the organism from the environment and may become infected with one or more strains which are primary resistant to the prophylaxis. Data for this come from studies of disseminated MAC where patients have been found to be infected with two or more genetically diverse strains concurrently causing so called polyclonal bacteraemia. ${ }^{3}$ These strains may have different drug sensitivity patterns including resistance to rifabutin. Obviously patients who are poorly compliant with prophylaxis may induce drug resistance. In the Nightingale study ${ }^{4}$ the drug susceptibility data were incomplete as only $59 \%$ of organisms were tested. Although the minimum inhibitory concentrations of rifabutin did not significantly differ between the placebo and treatment groups there is still discussion as to how to interpret these MIC data in terms of MAC resistance.

\section{Cost benefit}

In the USA the prevalence of MAC bacteraemia is approximately $5 \%$ at the time of AIDS diagnosis and then increases by approximately $20 \%$ per year. These data cannot be extrapolated to Europe as even within the USA there are large geographical variations. The maximum benefit of prophylaxis should be seen in countries where MAC incidence is highest and in the Nightingale study rifabutin reduced the risk of MAC bacteraemia by $50 \%$ although the absolute reduction was 9 cases per 100 taking the drug. The number of patients requiring to take rifabutin to produce a similar decrease in cases might be much higher in countries where MAC incidence is lower. The incidence for MAC in Europe is not known.

Interaction with tuberculosis

It is advised by the Center for Diseases Control ${ }^{5}$ that all patients who go on rifabutin prophylaxis should be screened for active tuberculosis (TB). Rifabutin has activity against $\mathrm{TB}$ and for many isolates cross resistance to rifampicin occurs and there is therefore the danger of TB monotherapy and subsequent drug resistance occurring. One such case has been recently reported. ${ }^{6}$ Such a screening policy causes several logistic problems. Firstly, in severely immune suppressed patients Mantoux testing is unreliable because of anergy. Secondly, chest radiographic changes of TB are often atypical. In patients who are from countries endemic for TB and have a high risk of reactivation, screening may need to be more regular as there are no data in humans to show that rifabutin prevents reactivation of TB. Patients who have a high risk of developing reinfection with TB may also need to be screened regularly for the same reason.

\section{Survival and quality of life}

So far there have been few data that suggests taking rifabutin prophylaxis has had a significant impact on overall survival. One study based on the Nightingale study suggest an $11 \%$ benefit. $^{7}$ To arrive at this figure many mathematical assumptions had to be made as survival was not an end point in the Nightingale study and subsequent follow up. Quality of life issues have not been addressed but those patients on prophylaxis who went on to develop breakthrough MAC bacteraemia did have a reduction in their symptoms at initial presentation. This is probably explained by the total mycobacterial load being lower than in those patients on placebo.

\section{Side effect and drug interactions}

Side effects of rifabutin are not a serious problem and uveitis usually occurs in those patients who are of a low body weight and are taking high doses (600 mg). Many patients with advanced HIV disease will be and are being offered multiple antiviral drugs. Rifabutin not only interacts with zidovudine but also leads to protease inhibitors not reaching therapeutic levels. Rifabutin also interacts with other drugs especially azoles and opiates. In severely immunosuppressed patients on polypharmacy these drug interactions have serious implications.

\section{Are there better alternatives?}

Some recent data suggest that clarithromycin might be a useful prophylaxis agent for MAC. However, drug resistance in those with breakthrough MAC may limit the use of this agent as a prophylaxis. Azithromycin has also been used in the prevention of MAC. In a study by the California Collaborative Treatment Group ${ }^{8}$ three arms of 220 patients each were randomised to azithromycin 1200 $\mathrm{mg}$ weekly, rifabutin $300 \mathrm{mg}$ daily or to a combination of these two drugs. On an intention to treat analysis it was shown that azithromycin alone was more efficacious than rifabutin, 31 compared with 52 breakthrough MACs and that the combination was also better than rifabutin. However, the combination did not cause a significantly greater reduction in breakthrough bacteraemia than azithromycin alone.

Prophylaxis for MAC bacteraemia may be more attractive using azithromycin and not only can it be taken 
weekly but it also has the added benefit that it is not related to any first line drugs used in TB. Its side effect and drug interaction profile is favourable. It has a price advantage as rifabutin daily costs approximately $£ 140$ per week and weekly azithromycin $£ 32$.

Should patients be offered MAC prophylaxis?

That depends on local epidemiology. Should patients be given rifabutin? That's for you to decide.

A POZNIAK

Department of Genitourinary Medicine,

Kings Healthcare NHS Trust

London SE5 9RS UK

1 Yajko DM, Chin DP, Gonzalez PC, Nassos PS, Hopewell PC, Reingold AL, et al. Mycobacterium avium complex in water, food and soil samples
collected from the environment of HIV infected individuals. $₹$ Acquired collected from the environment
Imm Defic Syndr 1995;9:176-82.

2 Maslow JM, Barber TW, Falkinham JO, Arbeit RD. Persistent colonisation of potable water as a source of Mycobacterium avium infection in AIDS. Lancet 1994;343:1137-41.

3 Arbeit RD, Slutsky A, Barber TW, Maslow JN, Niemczyk S, Falkinham $\mathrm{JO}$, et al. Genetic diversity among strains of Mycobacterium avium causing monoclonal and polyclonal bacteraemia in patients with AIDS. $f$ Infect Dis 1993;167:1384-90.

4 Nightingale SD, Cameron DW, Gordin FM, et al. Two controlled trials of rifabutin prophylaxis against Mycobacterium avium complex infection in rifabutin prophylaxis against Mycobact
AIDS. N Engl F Med 1993;329:828-33.

5 Ostroff SM, Speigle RA, Feinberg J, Benson CA, Horsberg CR. Preventing disseminated Mycobacterium avium complex disease in patients infected disseminated Mycobacterium avium complex disease in patients infec.
with human deficiency virus. Clin Infect Dis 1995;21(suppl 1):S72-6.

6 Bishai WR, Graham NMH, Harrington S, Page C, Moore-Rice K, Hooper $\mathrm{N}$, Chaisson RE. N Engl ₹ Med 1996;334:1573-6.

7 Moore RD, Chaisson RE. Survival analysis of two controlled trials of rifabutin prophylaxis against Mycobacterium avium complex in AIDS. AIDS 1995;9:1337-42.

8 Young LS. Treatment and prophylaxis of Mycobacterium avium complex. Inter f STS AIDS 1996;7(suppl 1):23-27.

Since the advent of the epidemic, the incidence of Mycobacterium avium complex (MAC) has steadily increased, until it now represents the commonest cause of bacterial infections in those living with the human immunodeficiency virus (HIV). ${ }^{12}$ Although the pathogenicity of MAC within the context of HIV disease was initially questioned, it has since been shown to be a cause of considerable morbidity and excess mortality. ${ }^{3-5}$

The true prevalence of MAC infection is unclear. Although studies have shown an incidence of approximately $25 \%$, autopsy studies showed that greater than $50 \%$ may be infected ${ }^{267}$ suggesting that in many cases the diagnosis is missed. Additionally, even when the diagnosis is made, almost $25 \%$ are after the patient has expired. ${ }^{4}$

It is clear that patients with fewer than $100 \mathrm{CD} 4 / \mathrm{mm}^{3}$ are at risk of disseminated disease, and that this risk increases as the CD4 count falls. ${ }^{3}$ Patients with less severe immunosuppression may present with localised disease, such as lymphadenitis, abscesses and other skin disease. ${ }^{89}$ In those patients with disseminated disease a wide variety of symptoms are seen, including malaise, fever, chills, weight loss, night sweats, abdominal pain, diarrhoea, nausea and vomiting. Examination is often unhelpful with nonspecific hepato-splenomegaly and lymphadenopathy.

Due to the excess morbidity and mortality seen with this disease, and due to the difficulties in diagnosis during life, and due to the fact that even when diagnosis is made there is no agreed therapy, in recent years, research has been focused on the prophylaxis of this condition. Despite the fact that evidence is now available that prophylaxis may reduce the incidence of this infection, as well as MAC related morbidity and mortality, there are still clinicians who fail to prescribe this prophylaxis. One is reminded of the early days of $\mathrm{HIV}$, when despite evidence that secondary prophylaxis was effective in reducing the recurrence of Pneumocystus carinii pneumonia (PCP), primary prophylaxis was still not given, leading to an incidence of this infection approaching $80 \% .{ }^{9}$

What is the evidence that MAC prophylaxis should be given? The best evidence for MAC prophylaxis (are the ADRIA studies from USA and Canada), showing that rifabutin reduces the incidence, morbidity and mortality of this infection. As the entry and exclusion criteria for these two studies were so similar, they are generally analysed together. ${ }^{10}$ Patients were eligible if their CD4 counts were less than $200 / \mathrm{mm},{ }^{3}$ and were receiving PCP prophylaxis and antiretroviral treatment, and had no evidence of any mycobacterial infection. Patients were randomised to receive either rifabutin $300 \mathrm{mg}$ once a day or placebo. After a mean of 239 days of follow up the study was stopped, having shown a significant reduction in the incidence of MAC in those receiving rifabutin.

In addition, this study showed a significant reduction in the incidence of symptoms associated with MAC in the rifabutin treated arm. There was an interesting difference between the two study groups as regards survival. Although the USA study showed a significant improvement in survival in those receiving rifabutin, a non-significant trend only was found in the Canadian study. On continued analysis there was no significant improvement in survival. This led many physicians not to give MAC prophylaxis. However, further follow up of this study by Chaisson and Moore showed not only a significant survival in those patients receiving rifabutin, but also an unexplained reduction in the incidence of both CMV disease and lymphoma. ${ }^{11}$ Another reason that physicians have been wary of giving rifabutin as MAC prophylaxis is the worry of resistance. There was no evidence that resistance was limited to the development of MAC in the rifabutin treated arm and breakthrough was probably due to either non-compliance or lack of absorption.

Another drug which has been shown to be effective against MAC prophylaxis is clarithromycin. In a study which has only been presented in abstract form, a reduction in the incidence of MAC occurred in patients receiving clarithromycin 500 bd rather than placebo. ${ }^{12}$ However in this study there was a high incidence of resistance in those developing breakthrough MAC on clarithromycin. Resistance may be reduced, and there also may be an increase in efficacy, by giving clarithromycin and rifabutin together, and this is presently being studied by the ACTG in the USA.

The third drug available for MAC prophylaxis is azithromycin. It has also been shown, in abstract form, to be effective in reducing the incidence of MAC infection, and appears to have the added advantage that once weekly dosing may be sufficient. ${ }^{13}$

In conclusion, despite the fact that prophylaxis is effective, not only in reducing the incidence of the disease but also an increase in survival from this condition, it is still not prescribed in some centres. The commonest reason now given for this is cost, which unfortunately is becoming one of the more important aspects in the choice of drug therapy. We must not be blindfolded to the expense of these drugs, as they may actually in the long run save money. This is well shown by Simpson's analysis of the original rifabutin study, in which she showed that patients who receive rifabutin cost less overall to care for, than those who did not, due to hospital admissions and cost of subsequent investigation and treatment. ${ }^{14}$

Chelsea and Westminster Hospital,

M R NELSON

369 Fulham Road,

London SW10, UK

1 Havlik JA Jr, Horsburgh CR, Metchock B, Williams PP, Fann A, Thompson SE, et al. Disseminated Mycobacterium avium complex infection; clinical identification and epidemiologic trends. $\mathcal{f}$ Infect Dis 1992;165:577-80.

2 Horsburgh CR. Mycobacterium avium complex infection in AIDS. $N$ Engl f Med 1991;324:1332-8. 
3 Nightingale SD, Byrd LT, Southern PM, Jockrusch JD, Cal CX, Wynne BA. Incidence of Mycobacterium avium-intracellulare complex bacteraemia in human immunodeficiency virus-positive patients. $\mathcal{F}$ Infect Dis 1992;165:1082-5

4 Chin DP, Reingold AL, Stone EN, Vittinghoff E, Horsburgh R, Simon $\mathrm{EM}$, et al. The impact of Mycobacterium avium complex bacteraemia and its treatment on survival of AIDS patients-a prospective study. 7 Infect Dis 1994;170:578-84.

5 Horsburgh CR Jr, Harlik JA, Ellis DA, Kennedy E, Fann SA, Dubois RE, et al. Survival of patients with acquired immune deficiency syndrome and disseminated Mycobacterium avium complex infection with and without antimycobacterial chemotherapy. Am Rev Resp Dis 1991;144:557-9.

6 Macher AM, Kovacs JA, Gill V, Roberts SD, Ames J, Park CH, et al. Bacteraemia due to Mycobacterium avium-intracellulare in the acquired Bacteraemia due to Mycobacterium avium-intracellulare in the

7 Reichert CM, O'Leary TJ, Levens DL, Simrell CR, Macher AM. Autopsy pathology in the acquired immunodeficiency syndrome. Am $\mathcal{F}$ Pathol 1983;112:357-82.

8 Inderlied CB, Kemper CA, Bermudez LEM. The Mycobacterium avium complex. Clin Microbiol Rev 1993;6:266-310.

9 Inwald D, Nelson M, Cramp M, Francis N, Gazzard B. Cutaneous mani- festations of mycobacterial infection in patients with AIDS. Brf Dermatol 1994;130:111-4.

10 Nightingale SD, Cameron DW, Gordin FM, Sullan PM, Cohn DL Chaisson RE, et al. Two controlled trials of rifabutin prophylaxis against Mycobacterium avium complex infection in AIDS. $N$ Engl $f$ Med 1993;329:828-33.

11 Moore RD, Chaisson RE. Survival analysis of two controlled trials of rifabutin prophylaxis against Mycobacterium avium complex in AIDS AIDS 1995;9:1337-42.

12 Pierce M, Crampton S, Henry D, Craft C, Nortario G. The effect of MAC and its Prevention on Survival in Patient with Advanced HIV Infection. In Abstracts of ICAAC 1995; San Francisco, USA.

13 Havllir DV, McCutchan JA, Bozzette SA, Dunne M. A double blind randomised study of weekly azithromycin and rifabutin for the prevention of Mycobacterium avium complex (MAC) in AIDS patients. Abstract 204; Mycobacterium avium complex (MAC) in AIDS patients. Abstract 204;
In: Abstracts of the 3rd Conference on Retroviruses and Opportunistic In: Abstracts of the 3rd

14 Simpson RN, Lavallee RL, Revicki DJ. Outcome and costs of short and long term rifabutin prophylaxis for Mycobacterium avium (MAC) disease in AIDS patients in the United Kingdom. AIDS 1994;8(54) Abstract 18.5. 